ARTICLE

Received 18 Feb 2015 | Accepted 1 Jul 2015 | Published 6 Aug 2015 DOl: 10.1038/ncomms8974 OPEN

\title{
Structural basis for drug-induced allosteric changes to human $\beta$-cardiac myosin motor activity
}

\author{
Donald A. Winkelmann', Eva Forgacs ${ }^{2}$, Matthew T. Miller ${ }^{3}$ \& Ann M. Stock ${ }^{3,4}$
}

Omecamtiv Mecarbil (OM) is a small molecule allosteric effector of cardiac myosin that is in clinical trials for treatment of systolic heart failure. A detailed kinetic analysis of cardiac myosin has shown that the drug accelerates phosphate release by shifting the equilibrium of the hydrolysis step towards products, leading to a faster transition from weak to strong actin-bound states. The structure of the human $\beta$-cardiac motor domain (cMD) with OM bound reveals a single OM-binding site nestled in a narrow cleft separating two domains of the human $\mathrm{CMD}$ where it interacts with the key residues that couple lever arm movement to the nucleotide state. In addition, OM induces allosteric changes in three strands of the $\beta$-sheet that provides the communication link between the actin-binding interface and the nucleotide pocket. The OM-binding interactions and allosteric changes form the structural basis for the kinetic and mechanical tuning of cardiac myosin.

\footnotetext{
${ }^{1}$ Department of Pathology and Laboratory Medicine, Robert Wood Johnson Medical School, Rutgers University, Piscataway, New Jersey 08854, USA.

${ }^{2}$ Department of Physiological Sciences, Eastern Virginia Medical School, Norfolk, Virginia 23507, USA. ${ }^{3}$ Center for Advanced Biotechnology and Medicine, Robert Wood Johnson Medical School, Rutgers University, Piscataway, New Jersey 08854, USA. ${ }^{4}$ Department of Biochemistry and Molecular Biology, Robert Wood Johnson Medical School, Rutgers University, Piscataway, New Jersey 08854, USA. Correspondence and requests for materials should be addressed to D.A.W. (email: winkelma@rwjms.rutgers.edu).
} 
eart failure is a common human disease with a significant lifetime risk that increases with age ${ }^{1}$. In its most common manifestation, heart failure is marked by a decrease in cardiac contractility culminating in systolic heart failure. Recently, a novel approach to the treatment of systolic heart failure has been developed on the basis of pharmacologic agents called 'cardiac myosin activators' that bind directly to myosin and target the kinetic mechanism driving contraction ${ }^{2}$. Directly targeting the contractile mechanism of cardiac myosin could theoretically improve heart performance without altering intracellular cAMP or calcium transients. A large, highthroughput, drug screen of a biochemically reconstituted contractile system identified a series of potential cardiac myosin activators $^{3}$. Omecamtiv Mecarbil (OM) is one such small molecule effector of cardiac myosin that is in clinical trials for treatment of systolic heart failure. Initial characterization of OM with bovine cardiac myosin shows that it binds directly to the myosin catalytic domain and operates by an allosteric mechanism to increase the transition rate of weak to strong bound actin states and enhance force generation ${ }^{4}$. In animal models, OM increases cardiac muscle function by extending the duration of systolic ejection without altering the heart rate ${ }^{4,5}$.

We have elucidated the precise action of OM on steady state and transient kinetics of the actomyosin mechanism and motor activity of porcine ventricular heavy meromyosin $(\mathrm{HMM})^{6}$. We find that OM shifts the equilibrium of the hydrolysis step of the myosin ATPase towards products, and this accelerates the flux through the product dissociation steps of the actomyosin mechanical cycle without altering the ADP dissociation rate, the key step that regulates the maximum shortening velocity ${ }^{7}$. The subtle changes in the kinetic mechanism lead to an increase in the transition rate of weak to strong bound actin states, resulting in increased number of force-producing crossbridges and, paradoxically, a dramatic reduction in the unloaded shortening velocity measured in vitro ${ }^{6,8}$. These fascinating kinetic results led to an intense effort to determine the drug-binding site and define the structural interactions within the human $\beta$-cardiac myosin motor domain that alter the motor activity.

Purification of human cardiac myosin was not an option for accomplishing this objective because of limited availability and poor stability of clinical specimens. The alternative, expression and purification of recombinant cardiac muscle myosin, had been technically unfeasible until we showed that the principal obstacle for the expression of vertebrate striated muscle myosin was that motor domain folding follows a regulated pathway that is unique to striated muscle cells ${ }^{9-12}$. To demonstrate this principle, we designed a striated muscle myosin motor domain (MD)::green fluorescent protein (GFP) chimeric protein and expressed it in muscle cells using adenovirus-mediated expression vectors ${ }^{9}$. This unique single-chain motor design is functionally active, fluorescent and ideally suited for structural studies.

Here we present the structure of the human $\beta$-cardiac motor domain (cMD) fused to GFP determined without drug (Apo structure) and with $\mathrm{OM}$ bound (OM + structure). OM binds in a narrow cleft that separates the $\mathrm{N}$-terminal $25-\mathrm{K}$ domain from the lower portion of the $50-\mathrm{K}$ domain of the cMD. The drug interacts with the key residues involved in coupling structural elements that are linked to the rotation of the lever arm into the pre-power stroke conformation. Strong binding to actin triggers the reversal of the rotation, leading to the power stroke and force production. Surprisingly, OM binding induces an allosteric change in the conformation of three strands of a $\beta$-sheet that are crucial to communication between the nucleotide pocket and actin-binding interface $^{13}$. Together, these interactions form the basis of the kinetic and mechanical effects of the drug. This structural analysis characterizes a highly significant binding cleft for drugs designed to modulate myosin motor activity.

\section{Results}

Human $\beta$-cardiac myosin motor domain design. The human $\beta$-cardiac myosin motor domain was expressed as a single-chain fusion protein with GFP. The protein design was based on a single-chain embryonic skeletal muscle MD::GFP chimera that was used to analyse motor domain folding ${ }^{9,10}$. The lever arm $\alpha$-helix projecting from the myosin MD is truncated within the IQ motif that forms the essential light-chain-binding site and joined to a short amino terminal $\alpha$-helix of GFP. This corresponds to residues $1-787$ of the human $\beta$-cardiac myosin fused to residues 5-238 of Aequorea victoria GFP. The chimeric gene was cloned into a replication-deficient adenovirus expression vehicle, and high-titre adenovirus stocks were used to infect post-mitotic $\mathrm{C} 2 \mathrm{C} 12$ myocytes. The brightly fluorescent cardiac MD::GFP protein was harvested from the myotubes and purified for crystallization. Nucleotide-free crystals of the $\beta$-cardiac MD::GFP were grown in hanging drops without OM (Apo structure) and with $125 \mu \mathrm{M}$ OM (OM + structure). SDS-polyacrylamide gel electrophoresis (SDS-PAGE) and tandem mass spectrometry (MS/MS) spectroscopy of the crystallized protein established that the full-length $117-\mathrm{kDa}$ cMD::GFP chimera was crystallized (Supplementary Fig. 1).

Structure of the Apo and $\mathrm{OM}+\mathrm{cMD}$. The structure of the cardiac MD::GFP chimera (cMD) was determined to a resolution of $3.2 \AA$ for the Apo and $2.25 \AA$ for the OM + crystals (Table 1). The drug-free Apo cMD crystallized in space group P1, and with $\mathrm{OM}$ bound, the protein crystallized in space group $\mathrm{P} 2_{1}$. There are two molecules (A and B chains) in the asymmetric unit in both crystal forms, and the overall conformation of the A and B chains in each structure is very similar, independent of drug. The protein was crystallized without nucleotide and is in the extended, nearrigour conformation. The folding pattern and domain structure of the Apo and OM + cMD structures are typical of the striated myosin II from chicken skeletal muscle root-mean-square deviation (r.m.s.d.) $=0.70 \AA$ for $558 \mathrm{C} \alpha$ atoms), scallop striated muscle (r.m.s.d. $=0.64 \AA$ for $544 \mathrm{C} \alpha$ atoms) and human $\beta$-cardiac myosin with AMPPNP bound ( $0.45 \AA$ for $608 \mathrm{C} \alpha$ atoms $)^{14-16}$ The domains and features of the cMD structure are illustrated for the A chain of the $\mathrm{OM}+$ structure in Fig. 1. The model is colourcoded to highlight the various regions of the structure associated with motor activity ${ }^{15,17,18}$.

The bound OM is nestled in a cleft and is just visible in the space-filling model (Fig. 1). The OM is more apparent in the ribbon diagram nestled between the $25-\mathrm{K}$ domain and the lower portion of the $50-\mathrm{K}$ domain. The $\mathrm{N}$-terminal $25-\mathrm{K}$ domain (blue) forms one face of the OM-binding cleft. About $35 \AA$ removed from the drug cleft the $25-\mathrm{K}$ domain also forms one face of the nucleotide-binding pocket. The lower $50-\mathrm{K}$ domain (red) includes major elements of the actin-binding surface and forms the other face of the OM-binding cleft. The upper 50-K domain (orange) includes additional elements of the actin-binding surface that are separated by a deep cleft from the lower $50-\mathrm{K}$ domain. The upper $50-\mathrm{K}$ domain forms the other surface of the nucleotide pocket. The converter domain (yellow) abuts the OM-binding cleft and couples the motor domain to the lever arm helix (LAH).

In all myosin II molecules, including $\beta$-cardiac myosin, the LAH forms the light-chain-binding domain; however, in this chimeric protein the helix was truncated in the centre of the essential light-chain IQ-binding motif and fused to a short N-terminal helix of GFP (residues 791-1026; green). As a consequence, the GFP domain is positioned by design in the 


\section{Table 1 | Data collection and refinement statistics.}

\begin{tabular}{|c|c|c|}
\hline & Apo cMD (4P7H)* & OM + cMD (4PAO) ${ }^{\dagger}$ \\
\hline \multicolumn{3}{|l|}{ Data collection } \\
\hline Space group & P1 & $\mathrm{P} 2_{1}$ \\
\hline \multicolumn{3}{|l|}{ Cell dimensions } \\
\hline$a, b, c(\AA)$ & 59.6, 97.7, 118.1 & $100.2,88.9,137.8$ \\
\hline$\alpha, \beta, \gamma\left({ }^{\circ}\right)$ & $71.0,82.4,75.0$ & $90.0,93.9,90.0$ \\
\hline Resolution ( $\AA$ ) & $25.0-3.2(3.3-3.2)^{\ddagger}$ & $46.0-2.25(2.33-2.25)$ \\
\hline$R_{\text {merge }}$ & 0.352 (1.299) & $0.258(1.367)$ \\
\hline$I / \sigma(I)$ & $9.0(2.0)^{\S}$ & $9.0(1.1)^{\S}$ \\
\hline Completeness (\%) & $99.5(98.0)$ & $93.3(84.1)$ \\
\hline Redundancy & $11.3(10.2)$ & $7.4(3.3)$ \\
\hline \multicolumn{3}{|l|}{ Refinement } \\
\hline Resolution $(\AA)$ & 3.2 & 2.25 \\
\hline No. of reflections & $39,840(3,966)$ & $107,033(9,608)$ \\
\hline$R_{\text {work }} / R_{\text {free }}$ & $0.191 / 0.265$ & $0.201 / 0.246$ \\
\hline \multicolumn{3}{|l|}{ No. of atoms } \\
\hline Protein & 14,882 & 14,533 \\
\hline Ligand/ion & 54 & 118 \\
\hline Water & 5 & 306 \\
\hline \multicolumn{3}{|l|}{$B$-factors } \\
\hline Protein & 49.0 & 46.0 \\
\hline Ligand/ion & 59.8 & 62.3 \\
\hline Water & 24.2 & 33.8 \\
\hline \multicolumn{3}{|l|}{ R.m.s.d. } \\
\hline Bond lengths $(\AA)$ & 0.007 & 0.011 \\
\hline Bond angles $\left({ }^{\circ}\right)$ & 1.08 & 1.35 \\
\hline \multicolumn{3}{|c|}{$\begin{array}{l}\text { cMD, } \beta \text {-cardiac motor domain; OM, Omecamtiv Mecarbil; r.m.s.d., root-mean-square deviation. } \\
\text { *Data collected from three crystals. } \\
\text { tData collected from a single crystal. } \\
\text { fValues in parentheses are for the highest-resolution shell. } \\
\text { \$The Apo data set was truncated at } 3.2 \AA \text { with the mean }(I / \sigma(I))=2 \text { and a CC* }>0.88 \text { for the highest-resolution shell }{ }^{61} \text {. The } C C^{*} \text { statistic for the OM }+2.33-2.25 \AA \text { resolution shell is }>0.73 \text {, supporting } \\
\text { the inclusion of the full data range in determination of the model }{ }^{62,63} \text {. Applying traditional cutoff criteria of the mean }(I / \sigma(I)) \geq 2 \text { would make this a nominal } 2.40-\AA \text {-resolution structure. }\end{array}$} \\
\hline
\end{tabular}

space normally occupied by the light chains ${ }^{9}$. The GFP chromophore occupies a cavity in the centre of the GFP $\beta$-barrel. modulating the activity corroborate the specificity of the binding site observed in the crystal structure.
Effect of OM on cardiac myosin motor activity. The GFP domain substitutes a rigid $\beta$-barrel for the myosin light chains and provides a convenient handle for assaying the effect of drug binding on cMD motor activity by using anti-GFP to immobilize the cMD on surfaces ${ }^{9}$. The unloaded shortening velocity was measured using the in vitro motility assay (Fig. 2). The cMD::GFP chimera supports sliding actin filament velocities of $0.8 \mu \mathrm{m} \mathrm{s}^{-1}$, equivalent to the human $\beta$-cardiac HMM (cHMM) and comparable to the velocity measured for porcine ventricular HMM $\left(0.96 \mu \mathrm{m} \mathrm{s}^{-1}\right)^{6}$. Addition of OM to the assay dramatically slows the filament velocity (15-20-fold) powered by both the cMD and cHMM. This is identical to what was found with porcine ventricular HMM and is explained by a drug-induced increase in crossbridges interacting with the actin filaments that impose an internal load on the sliding filament movement ${ }^{6,8}$. The $K_{\mathrm{i}}$ for OM inhibition of the sliding velocity powered by the cMD is $100 \mathrm{nM}$, indicating a high affinity for the intermediates of the mechanochemical cycle. The sliding filament velocity powered by a fast skeletal muscle myosin is much faster $\left(6.3 \mu \mathrm{m} \mathrm{s}^{-1}\right)$ than the cMD, as is expected, but is virtually unaffected by the drug, consistent with the report that $\mathrm{OM}$ does not bind to skeletal muscle myosin ${ }^{4}$.

OM modulates the steady-state ATPase of the cMD, decreasing the $V_{\max }$ from 5.3 to $1.9 \mathrm{~s}^{-1}$ but also lowering the $\mathrm{K}_{\mathrm{ATP}}$ ase for actin activation fourfold from 22.5 to $5.4 \mu \mathrm{M}$ (Supplementary Fig. 2). This is consistent with the effect of OM on the porcine ventricular HMM ATPase activity. The effects of OM on the cMD motor activity and ATPase activity and the 100-nM $K_{\mathrm{i}}$ for
OM-binding site and the coupling region. The drug, $\mathrm{OM}$, is tightly nestled in a narrow cleft separating the lower 50 -K domain from the N-terminal $25-\mathrm{K}$ domain (Fig. 3 and Supplementary Fig. 3). OM is in an extended conformation that lies along the SH1 helix (698-707) and is adjacent to C705 (SH1) on one side and extends to and interacts with the first strand (711-713) of the three-stranded $\beta$-sheet of the converter domain. The drug packs against and wraps around two loops from the $25-\mathrm{K}$ domain. $\mathrm{OM}$ binding involves extensive interaction with at least six residues from the 25-K domain (A91, M92, L96, S118, G119 and F121), two from the relay helix (M493 and E497), four from the SH1 helix (V698, G701, I702 and C705) and four from the $\beta$-sheets of the converter domain (P710, N711, R712 and L770; Supplementary Table 1). There are four potential hydrogenbonding interactions between the protein and drug that involve A91, M92, S118 and N711. The N711 H-bond to the OM methyl-pyridine ring is of interest because this residue is a serine in fast skeletal muscle myosin and is one of only three sequence differences in the binding cleft that might account for the selectivity of the drug for modulating the activity of cardiac myosin but not skeletal myosin (Supplementary Table 2). The same interactions are found in the protein-drug-binding site of the $\mathrm{A}$ and $\mathrm{B}$ chains of the $\mathrm{OM}+$ structure, although the conformation of the drug and binding cleft is slightly different in the two molecules. The A chain-binding site is depicted in Fig. 3.

The conformation of the main chain and the side-chain rotamers of residues lining the drug-binding cleft are essentially the same in the Apo and OM+ structures, indicating that drug 
a

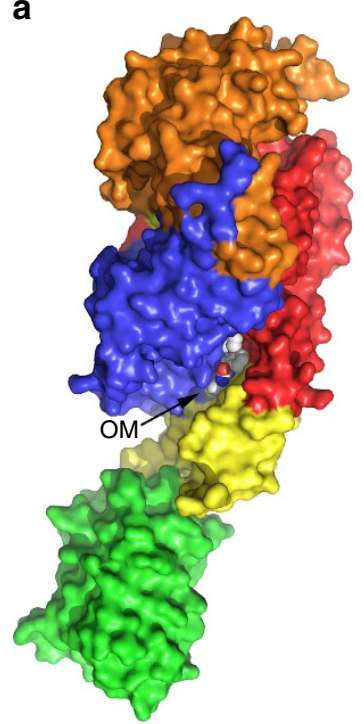

b

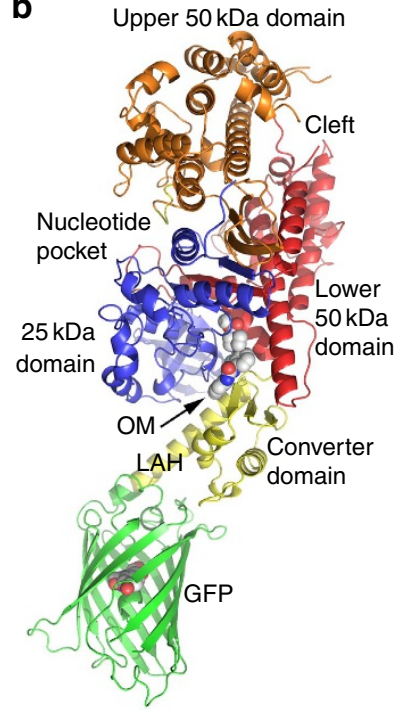

Figure 1 | The structural organization of the human $\beta$-cardiac MD with bound $\mathbf{O M}$. (a) Space-filling model of the A chain of the OM+ structure showing the buried OM-binding site nestled between the N-terminal 25-K domain (residues 2-204; blue) and the lower 50-K domain (residues 471-708; red). The upper 50-K domain (211-470; orange) is separated by the 'Cleft' from the lower $50-\mathrm{K}$ domain. There is no nucleotide in this structure, the cleft is 'open' and the lever arm is extended consistent with the near-rigour conformation. The converter domain (residues 709-777) and $\mathrm{LAH}$ (residues 778-787) are in yellow and the GFP domain is in green. (b) Ribbon diagram of the same orientation to show the OM-binding site bound deeply in a narrow cleft between the $25-\mathrm{K}$ and lower $50-\mathrm{K}$ domains. The LAH linking the converter domain to the GFP domain is labelled. The conformation of the Apo CMD structure is very similar to the OM+ structure shown here with an r.m.s.d. $=1.14 \AA$ for all $962 \mathrm{C} \alpha$ atoms.

binds to an existing cleft and does not induce significant local perturbation of the $\mathrm{CMD}$ conformation. However, analysis of the protein-drug interface indicates that $\mathrm{OM}$ binding results in extensive solvent shielding of residues that couple the structural elements that are central to the conformation changes of the recovery stroke: the $\mathrm{SH} 1 \mathrm{helix}$, the relay helix and the converter domain. Photo-crosslinking of a benzonphenone derivative of OM had identified this cleft as the potential OM-binding site ${ }^{4}$. The drug-binding site is consistent with that crosslinking experiment; however, the drug is deeper in the cleft than previously imagined.

The recovery stroke involves the priming of the lever arm at the start of the mechanical cycle resulting in the rotation of the converter domain and LAH through $\sim 65^{\circ}$. The priming step is a conformational equilibrium driven by the nucleotide bound in the active site. The nucleotide state is transmitted via the relay helix to a coupling region that links the SH1 helix, relay helix and converter domain together. The packing of the drug against the SH1 helix, relay helix and converter domain shields these elements from solvent and enhances interactions among them ${ }^{19}$. For example, OM buries the only solvent-accessible surface of the E497-R712 salt bridge that links the relay helix to the converter domain, thereby fortifying the bond. It also shields the M493C705 H-bond that links the relay helix to the SH1 helix (Fig. 3).

Converter domain-LAH-GFP rotation. There are two distinct molecules in the unit cell (A and B) of both the Apo and OM+ structures. In both structures the core motor domains (residues

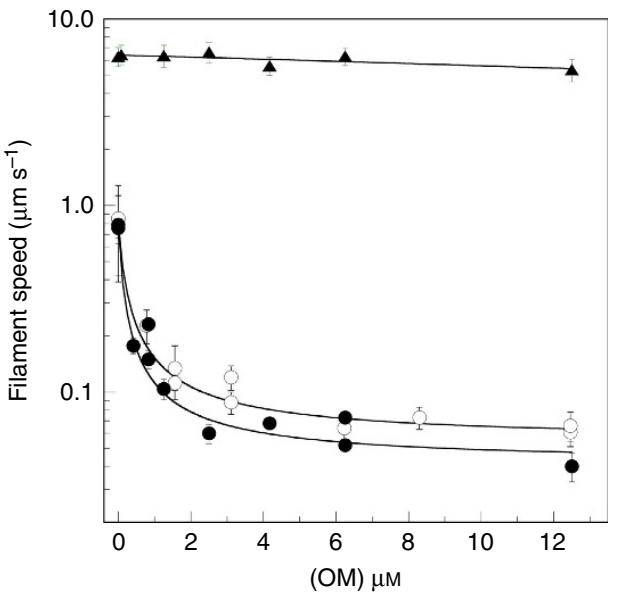

Figure 2 | The unloaded shortening velocity of cMD is inhibited by $O M$. Titration of the effect of OM on the unloaded shortening velocity measured with the in vitro motility assay. The CMD was bound indirectly to surfaces through an anti-GFP monoclonal antibody $(\bullet)$ and compared with the human $\beta$-cardiac HMM $(O)$ bound directly to the nitrocellulose surface. In the absence of the drug, both cardiac myosin fragments support actin filament velocities of $0.8 \mu \mathrm{ms}^{-1}$. Titration with $\mathrm{OM}$ from 0.42 to $12.5 \mu \mathrm{M}$ dramatically slowed the velocity of filament movement by $15-20$-fold. The apparent $K_{\mathrm{i}}$ for OM binding is $100-150 \mathrm{nM}$ for the human $\mathrm{CMD}$ and $\mathrm{cHMM}$. Adult fast skeletal muscle myosin $(\boldsymbol{\Delta})$ moves actin filaments at $6.3 \mu \mathrm{ms}^{-1}$ and even the highest concentration of $\mathrm{OM}$ tested has little effect on filament velocity. Assays were conducted at $32^{\circ} \mathrm{C}$. The cardiac motor activity was fit to the hyperbolic inhibition curve: $v_{0}=\frac{V_{\max }-\left(V_{\max }-V_{i}\right) \times[\mathrm{OM}]}{K_{i}+[\mathrm{OM}]}$; where, $v_{0}$ is the observed velocity, $V_{\max }$ is the unloaded shortening velocity, $V_{i}$ is the maximum inhibited velocity and $[\mathrm{OM}]$ is the concentration of drug. The mean velocity \pm s.d. of the velocity distribution for hundreds of filaments is plotted.

2-708) are packed tightly together in the crystal lattice with extensive non-crystallographic symmetry between them. The conformation of the core motor domain is very similar in the two molecules of the Apo (r.m.s.d. $=0.305 \AA$ for $567 \mathrm{C} \alpha$ atoms) and the two molecules of the $\mathrm{OM}+$ (r.m.s.d. $=0.311 \AA$ for $550 \mathrm{C} \alpha$ atoms) structures. The similarity is shared between corresponding core motor domains of the Apo and $\mathrm{OM}+$ in pairwise comparison (Apo-A to OM-A r.m.s.d. $=0.33 \AA \AA$; Apo-B to OM-B r.m.s.d. $=0.36 \AA$ ). The organization of the converter domains, the position of the GFP domains and the LAH linking the two are markedly different between the A and B chains in the asymmetric unit of both crystal structures. There is an $\sim 15^{\circ}$ rotation of the converter domain-LAH-GFP domain as a unit between the two molecules in the unit cell of both crystal structures (Fig. 4). The apparent $15^{\circ}$ rotation of the $\mathrm{LAH}$ from the $\mathrm{A}$ to the $\mathrm{B}$ position is in the same direction as a much larger LAH rotation during the recovery stroke $\left(\sim 65^{\circ}\right)$. The conformation change involves movement of the relay helix and a portion of the N-terminal $25-\mathrm{K}$ region called the SH3 domain in addition to the converter domain-LAH. The hinge point for the rotation is G708 of the cMD, adjacent to the OM-binding cleft. As a consequence, the conformation of the drug-binding cleft is different between the $\mathrm{OM}+\mathrm{A}$ and $\mathrm{B}$ chains.

OM moves with the rotation of the converter domain. The changes in the drug cleft as a consequence of the $15^{\circ}$ rotation of the converter domain-LAH in the two molecules of the OM+ structure (OM-A and OM-B) are shown in Fig. 5. To accommodate changes in the position of the converter domain, there is 


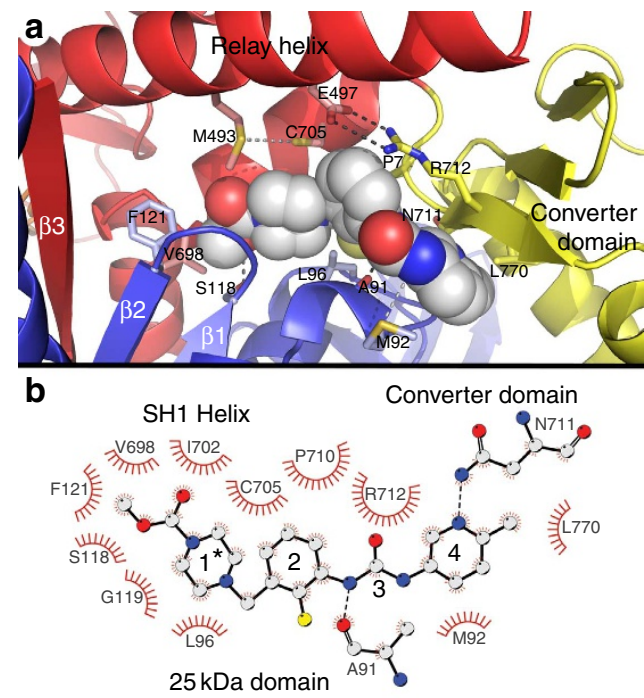

Figure 3 | OM-binding site on the human $\boldsymbol{\beta}$-cardiac MD. (a) A ribbon diagram of the A chain of the $\mathrm{OM}+$ structure is shown with OM (spacefilling model) nestled in a narrow cleft separating the $25-\mathrm{K}$ domain (blue) from the lower 50-K domain (red), and interacting with residues from these domains and the converter domain (yellow). The drug is in an extended conformation with the carboxymethyl-piperazine group buried deeply in the cleft. One surface of this ring and the fluoro-benzene ring of OM make extensive packing interactions with residues along one face of the $\mathrm{SH} 1$ helix including $\mathrm{C} 705$ ( $\mathrm{SH} 1$ ). The drug bridges the gap from the $\mathrm{SH} 1$ helix to the first strand (N711-1713) of the three-stranded $\beta$-sheet of the converter domain. In bridging the gap, OM buries a bi-dentate salt bridge, E497-R712, beneath the fluoro-benzene ring. The amido group of N711 forms a H-bond with the nitrogen of the OM methyl-pyridinyl ring. The other surface of OM wraps around a turn (A91-L96) of the 25-K domain and interacts with the first two strands ( $\beta 1-\beta 2)$ and connecting loop of the seven-stranded $\beta$ sheet of the CMD. The 25-K domain residues S118, A91 and M92 form likely $\mathrm{H}$-bonds with OM (shown with dotted lines), and there are at least 12 packing and hydrophobic interactions as well ${ }^{19}$. (b) A protein-drug interaction diagram ${ }^{64}$ summarizing the most extensive interactions in the binding cleft. Hydrogen bonds involving A91 and N711 are indicated in the diagram; however, two additional $\mathrm{H}$-bonds between OM and M92 and S118 are not drawn in the diagram for clarity. All of the interactions between OM and the binding cleft residues are summarized in Supplementary Table 1. *Numbers associated with the drug are added to identify the subregions of the molecule: (1) carboxymethyl-piperazine ring; (2) fluoro-benzine ring; (3) amino-carbamoyl linker; and (4) methyl-pyridinyl ring. The drug-binding interactions and the accessible and buried surface areas of the residues in the binding cleft were analysed with PISA (Protein Interfaces, Surfaces and Assemblies) ${ }^{19}$.

rotation of the fluoro-benzene and methyl-pyridinyl rings and the amino-carbamoyl linker of OM. These changes mirror the shift in position of the residues interacting with the drug. In particular, the bond distance for the H-bond with N711 remains constant despite significant movement of the $\beta$-strands of the converter domain. OM follows the linkage between the SH1 helix and converter domain-LAH elements in the two molecules (OM-A and $\mathrm{OM}-\mathrm{B})$. The carboxymethyl-piperazine ring of $\mathrm{OM}$ is most deeply buried in the drug-binding cleft and changes very little between the two conformations of the cleft. This OM ring interacts with residues of strands $\beta 1$ and $\beta 2$ of the central sevenstranded $\beta$-sheet of the cMD structure. Comparison of the Apo and $\mathrm{OM}+$ structures reveals subtle changes in the seven-stranded $\beta$-sheet that propagate out from the drug-binding cleft, perhaps arising from the interaction with these first two strands.
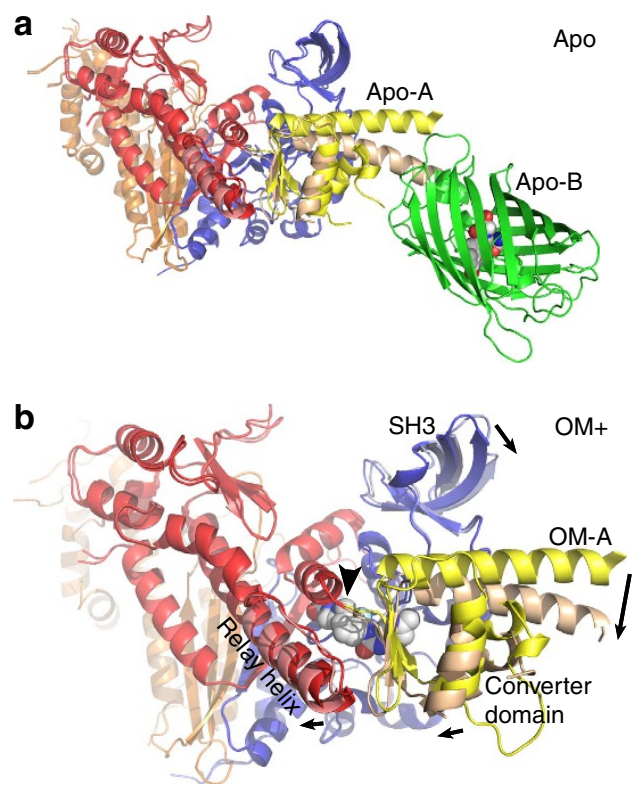

Figure 4 | The two cardiac MD::GFP molecules in the asymmetric unit for both the Apo and the OM + structures differ in the rotation of the LAH and GFP. In both structures, the B chain converter domains, LAH and GFP, are rotated $\sim 15^{\circ}$ from the near-rigour configuration towards the pre-power stroke state. (a) The core MD (residues 2-708) of the Apo-A and B chains is aligned to show the rotation of the $B$ chain converter domain and $L A H$ (coloured tan). The A chain GFP domain is not shown for clarity. (b) The A and $B$ chains of $O M+$ structure are similarly aligned and enlarged to show the rotation of the $\mathrm{LAH}$. The $\mathrm{A}$ chain $\mathrm{OM}$ molecule is shown as a spacefilling model in the centre of the image. The $B$ chain converter domain and $\mathrm{LAH}$ are coloured tan. The arrows indicate the direction of rotation of the SH3-like domain, the $\mathrm{LAH}$, the converter domain and the relay helix of the $\mathrm{B}$ chain relative to the $A$ chain. The point of rotation in both structures is G708, immediately above OM and marked by an arrowhead in $\mathbf{b}$. Perhaps as a consequence of the rotation, the $\mathrm{B}$ chain converter domains, $\mathrm{LAH}$ and GFP, are less well defined in both structures with higher crystallographic atomic displacement parameters.

The transducer region. Twisting and distortion of the last three strands $(\beta 5, \beta 6$ and $\beta 7)$ of the seven-stranded $\beta$-sheet of the myosin $\mathrm{MD}$ is essential for rearrangements within the nucleotidebinding pocket depending on the nucleotide state and forms the structural basis for communication between the actin-binding interface, the nucleotide pocket and the converter domain ${ }^{13,20}$. The structural elements have been called collectively 'The Transducer' and include Loop 1 (204-210), the $\beta$-bulge (252-259) connecting strands $\beta 6$ and $\beta 7$ and the loop-linking helix $\mathrm{O}(\mathrm{HO})$ to the $\beta 5$-strand (448-455). The $\beta 5$-strand leads directly into Switch II (SW-II): the $\gamma$-phosphate-sensing loop in the nucleotide pocket. Switch II in turn leads into the relay helix, the coupling region and the drug-binding cleft.

OM interactions within the binding cleft include residues of the strands $\beta 1$ and $\beta 2$ of the central seven-stranded $\beta$-sheet. Comparison of the Apo and $\mathrm{OM}+$ structures reveals changes in the $\beta$-sheet that propagates out from the drug-binding cleft and results in a twist of the final three strands $(\beta 5, \beta 6$ and $\beta 7)$ of the sheet affecting the $\beta$-bulge and the HO- $\beta 5$ loop, key elements of the Transducer region (Fig. 6). The conformations of the two molecules in the OM+ structure (OM-A and OM-B) are not identical to one another in this region (Fig. 6a,b). The OM-B conformation has a greater twist, an extension of the $\beta 6$ and $\beta 7$ strands of the sheet into the $\beta$-bulge, and displacements of $1-3 \AA$ in the $\mathrm{C} \alpha$ positions for residues of the $\beta$-bulge and the HO- $\beta 5$ 


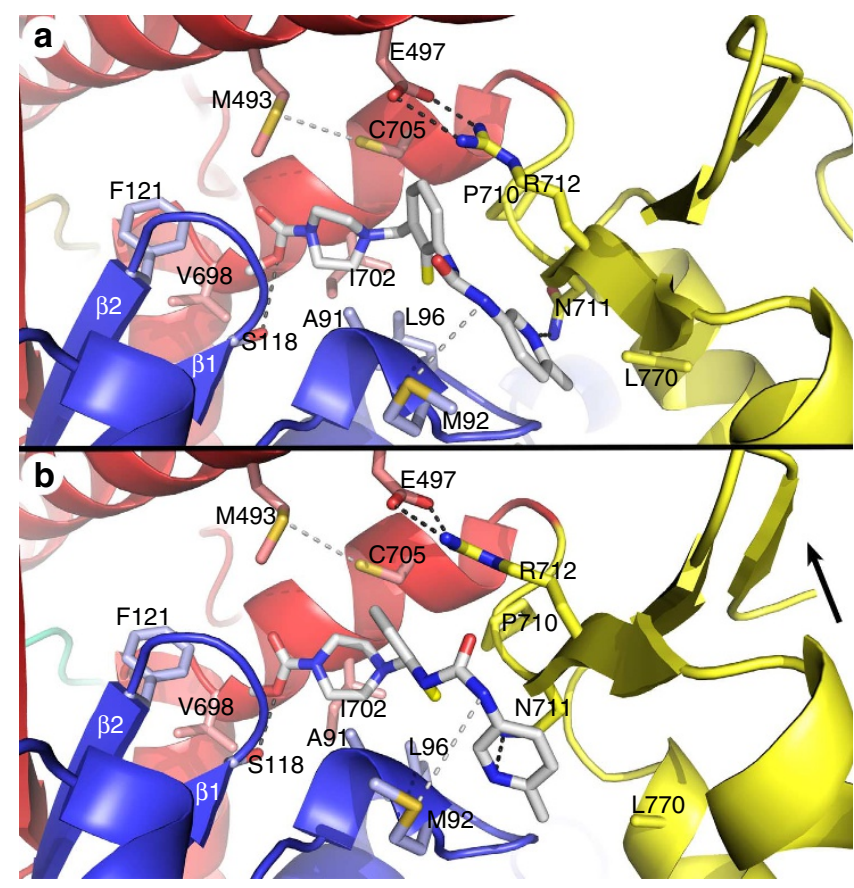

Figure 5 | The differences in the drug-binding cleft between the two molecules in the $\mathbf{O M}+$ structure. (a) The A chain conformation of the drug-binding site showing the interacting residues. (b) The $B$ chain conformation of the drug-binding site. OM interacts with the same set of residues in both conformations of the binding cleft. The principal change between $\mathrm{OM}-\mathrm{A}$ and $\mathrm{OM}-\mathrm{B}$ is the rotation of the converter domain indicated by the arrow in $\mathbf{b}$. In this view the converter domain moves up and towards the viewer, shifting the position of both N711 and R712. The fluoro-benzyl and methy-pyidinyl rings of OM rotate between OM-A and OM-B conformations as does the amino-carbamoyl group linking them. This maintains the H-bond to N711 and the shielding of the E497-R712 salt bridge. The carboxymethyl-piperazine is buried deep in the cleft and interacts with S118 and F121 of the $\beta 1$ and $\beta 2$ strands of the seven-stranded $\beta$-sheet.

loop. There also is subtle shift at the transition of $\beta 5$-strand into SW-II (arrow in Fig. 6b). The conformations of these elements in both molecules of the Apo structure are essentially identical and differ from both molecules in the $\mathrm{OM}+$ structure (Fig. 6c). The nucleotide-free conformation of the Transducer region of the OM-B molecule is in fact more similar to that in the cardiac myosin MD with AMPPNP bound ${ }^{14}$ than either Apo structure or the OM-A molecule in the same unit cell (Supplementary Fig. 4b).

The crystallographic packing between the two molecules of the Apo and the $\mathrm{OM}+$ structures is essentially identical and does not involve the elements of the Transducer region, suggesting that crystal packing is not the source of this highly localized conformational change. In addition, this region of the structure is well defined in both the Apo and OM + structures with B-factors that are about half of the average for the protein as a whole. All of these changes are $\sim 35 \AA$ distant from the OM-binding site and thus are allosteric changes affecting a link in the communication between the nucleotide pocket and the actin-binding interface.

We conclude that the stabilization of the coupling between the SH1 helix, relay helix and converter domain within the drug-binding cleft and the allosteric distortion of the $\beta$-sheet in the Transducer region are the principal structural changes associated with drug binding. These changes may be sufficient

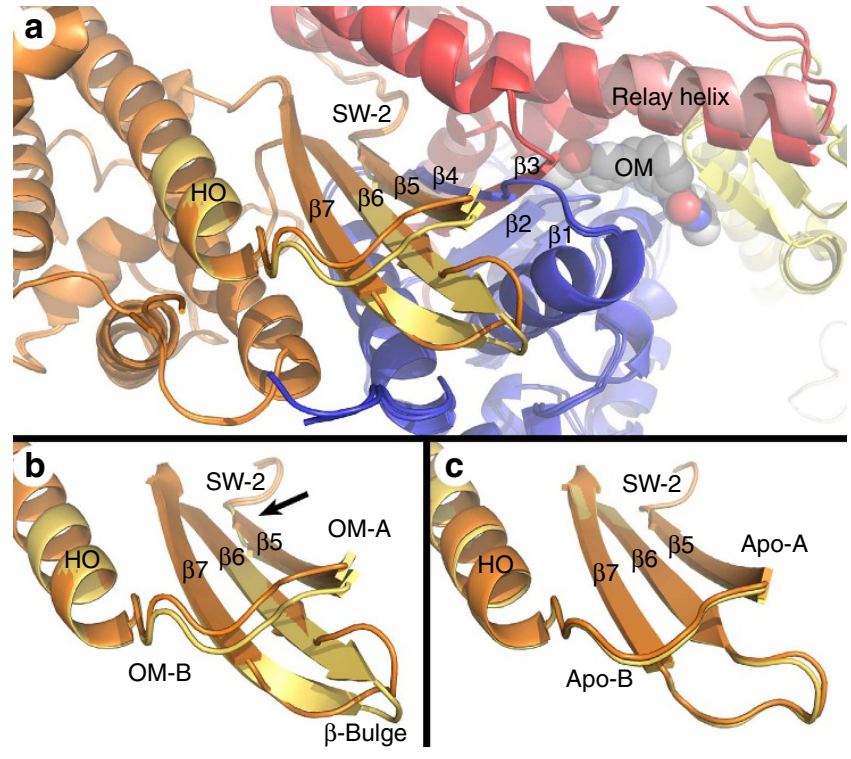

Figure 6 | Conformational changes in the Transducer region in the OM + structure. (a) The core CMD (2-708) of the OM-A and OM-B chains were aligned. Overall, the conformations of the two chains are nearly identical (r.m.s.d. $=0.31 \AA$ ); however, strands $\beta 5, \beta 6$ and $\beta 7$ of the $\beta$-sheet and the elements of the Transducer region (the $\beta$-bulge and HO- $\beta 5$ loop) are significantly different. The OM-A chain in this region is coloured orange and the OM-B chain is yellow-orange. Displacement of the relay helix as a result of $\mathrm{LAH}$ rotation is also highlighted. Note that OM (space-filling model) in the binding cleft is $35 \AA$ from the Transducer elements. (b) Comparison of the isolated Transducer elements of OM-A (orange) and OM-B (yellow-orange) showing an increased twist, a downward shift of the HO- $\beta 5$ loop and extension of the $\beta 6$ and $\beta 7$ strands of the sheet into the $\beta$-bulge. The arrow highlights the changes in $\beta 5$ to SW-II link. These differences are between the two molecules in the same unit cell. (c) In contrast, the Apo-A (orange) and Apo-B (yellow-orange) transducer elements superimpose. The Transducer region changes shown here are not as large as those reported for cleft closure as seen in myosin $\mathrm{V}$ (Supplementary Fig. 4b) but are following the same trajectory.

to account for the shift in the hydrolysis equilibrium in the nucleotide pocket and the accelerated rate of $\gamma$-phosphate release contributing to the measured kinetic effects of drug binding on cardiac myosin 6 .

\section{Discussion}

Here we report the structural basis for the allosteric mechanism of the novel cardiac myosin-specific drug, OM. The drug binds to the human $\beta$-cardiac myosin motor domain in a narrow cleft between the $\mathrm{N}$-terminal $25-\mathrm{K}$ domain and the lower portion of the $50-\mathrm{K}$ domain. Within the binding cleft the drug is intimately involved with the key elements of the recovery stroke. In addition, $\mathrm{OM}$ binding induces allosteric changes distant from the binding cleft that affect the twist of the central seven-stranded $\beta$-sheet of the motor domain. These interactions help explain how the drug modulates cardiac myosin motor activity.

In addition to OM, there are several other reversible allosteric effectors of myosin activity that bind to the motor domain including blebbistatin, halogenated pseudilins and EMD 57033. Blebbistatin binds at the apex of the myosin cleft separating the upper and lower $50-\mathrm{K}$ domains blocking the myosin in a product complex with low actin affinity ${ }^{21,22}$. The halogenated pseudilins are a class of myosin inhibitors with activity similar to blebbistatin that also bind in the cleft but at a site that is $\sim 7.5 \AA$ 
from the blebbistatin site ${ }^{23,24}$. EMD 57033 is a thiadiazinone derivative that stabilizes myosin against thermal denaturation and is an allosteric activator of $\beta$-cardiac myosin ATPase and myofilament contraction ${ }^{25}$. In silico docking studies suggest that it binds in a pocket near the N-terminal SH3 domain. None of these other allosteric effectors bind in or near the OM cleft or are under consideration for therapeutic potential.

The key to the actomyosin mechanochemical cycle is the ability of the myosin motor domain to couple small changes in the catalytic ATPase site to large conformational changes in both the actin-binding and force-generating domains ${ }^{26}$. This pathway is summarized in the actomyosin kinetic scheme in Fig. 7. The nucleotide bound in the active site (ATP, ADP $\cdot \mathrm{P}_{\mathrm{i}}$ or ADP) defines the conformation of the MD and its affinity for actin. This requires a communication pathway to amplify changes in the nucleotide pocket and couple them to domain movements. $\mathrm{OM}$ acts along this pathway as a traditional allosteric effector binding $\sim 35 \AA$ from the nucleotide pocket and altering the ATP hydrolysis mechanism and motor activity of cardiac myosin ${ }^{4,6,27}$. A detailed analysis of the transient kinetics of porcine ventricular HMM reveals that OM does not activate of the actomyosin ATPase activity as was originally suspected ${ }^{4}$ and instead inhibits the $V_{\max }$ by two- to threefold ${ }^{6}$. This was shown here for the human $\beta$-cardiac myosin as well. The principal effect of $\mathrm{OM}$ on the myosin kinetic mechanism is a shift in the equilibrium for ATP hydrolysis in the active site towards product formation $\left(\mathrm{M} \uparrow \cdot \mathrm{ADP} \cdot \mathrm{P}_{\mathrm{i}}\right)$ that is coupled to an increase in the rate of the fast phase of phosphate release. These changes produce an increase in

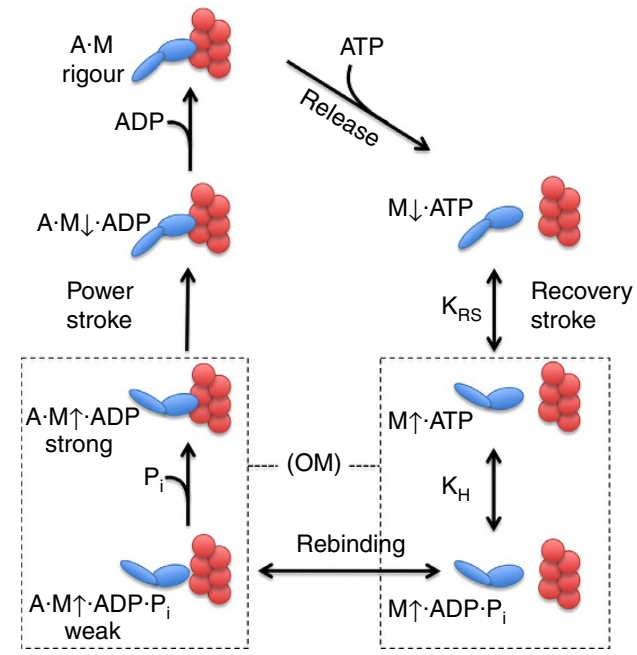

Figure 7 | A general kinetic scheme for the actomyosin ATPase cycle. The myosin motor domain ( $M$, blue) is shown at the top left at the end of the cycle, tightly bound to actin ( $A$, red) in the nucleotide-free rigour state $(A \cdot M)$. ATP binds rapidly and releases the MD from actin. The MD then undergoes a conformational equilibrium before ATP hydrolysis between two states designated as $M \downarrow$ and $M \uparrow$. These states correspond to the nearrigour $(M \downarrow)$ and pre-power stroke $(M \uparrow)$ conformations. The arrows indicate the down position of the LAH $(M \downarrow)$ and the up position in the pre-power stroke state $(\mathrm{M} \uparrow)$ and correlate with intrinsic fluorescent states. The 'Recovery Stroke' is the rapid equilibrium $\left(K_{\mathrm{RS}}\right)$ between these states. The hydrolysis equilibrium $\left(K_{\mathrm{H}}\right)$ is coupled to this transition before binding to actin ${ }^{6}$. On rebinding to actin the weak to strong transition is coupled to $\gamma$-phosphate $\left(P_{i}\right)$ release. This results in a conformation change triggering rotation of the LAH producing the 'Power Stroke' and force production. Rapid ADP dissociation follows, completing the cycle. OM influences the hydrolysis equilibrium $\left(K_{H}\right)$, and it accelerates $P_{i}$ release associated with the weak to strong actin-binding transition ${ }^{6}$. These steps are boxed with dotted lines. the flux of the intermediates through the actomyosin mechanochemical pathway leading to a faster transition of weak to strong bound actin states. In the kinetic assays, we measured the unloaded rate of ADP dissociation from actomyosin ADP at the end of the contractile cycle and showed that OM does not affect this step. Faster phosphate release without a change in ADP dissociation leads to a higher fraction of myosin bound to actin in a strong $\mathrm{A} \cdot \mathrm{M} \uparrow \cdot \mathrm{ADP}$ state ${ }^{6}$.

OM produces a large (15-20-fold) reduction in the unloaded shortening velocity measured in the in vitro motility assay (Fig. 2). The reported $K_{\mathrm{i}}$ reflects the apparent affinity of OM for a composite of steps in the kinetic pathway rather than a binding constant for any single step. Specifically, OM affects both the hydrolysis equilibrium $\left(\mathrm{M} \uparrow \cdot \mathrm{ATP}\right.$ to $\left.\mathrm{M} \uparrow \cdot \mathrm{ADP} \cdot \mathrm{P}_{\mathrm{i}}\right)$ and $\mathrm{P}_{\mathrm{i}}$ release $\left(\mathrm{AM} \uparrow \cdot \mathrm{ADP} \cdot \mathrm{P}_{\mathrm{i}}\right.$ to $\mathrm{AM} \uparrow \cdot \mathrm{ADP}$ transitions). Thus, the measured $K_{\mathrm{i}}$ reflects the interaction of $\mathrm{OM}$ with an ensemble of states. The effect on the motility assay is likely a result of the recruitment of crossbridges to the actin filament as a consequence of the faster transition from weak to strong bound actin states, resulting in a higher fraction of cMDs tightly bound to actin producing an internal drag on filament movement. Although this slows the velocity of filament movement, it also leads to more force production per filament, a favourable outcome for improving cardiac systolic function. A strain-dependent slowing of the ADP dissociation rate has recently been reported for porcine $\beta$-cardiac myosin, and this additional mechanism could contribute to the decrease in filament velocity ${ }^{28}$. A strain-dependent step would extend the duration of systole as is reported in animal models of heart failure treated with $\mathrm{OM}^{5}$. An increase in strongly attached crossbridges induced by $\mathrm{OM}$ also could facilitate systolic $\mathrm{Ca}^{2+}$ activation in the heart by triggering movement of the Tpm-Tn switch out of the inhibitory state. However, the significance of this activation mechanism under physiological conditions in heart muscle has been questioned recently ${ }^{29,30}$

The communication pathway linking the ATPase state to the rotation of the lever arm has been delineated by crystal structures of non-cardiac myosin motor domains with different bound ATP analogues ${ }^{13,18,31,32}$. These structures reveal distinct orientations of the converter domain that lead to an $\sim 65^{\circ}$ rotation of the LAH. On the basis of these known structural states, mutational analyses and molecular dynamics simulations, it has been proposed that with ATP bound the myosin motor domain exists in two alternative conformations, the pre-recovery $\mathrm{M} \downarrow$ state (down state) and post-recovery $M \uparrow$ state (up state with rotated $\mathrm{LAH})^{33-37}$. The transition between these myosin conformations is controlled by the myosin nucleotide interaction and is called the recovery stroke. The pre-recovery state $(\mathrm{M} \downarrow)$ has nucleotide bound with SW-II in the open position. In the $\mathrm{M} \uparrow$ state the SW-II loop closes and a glycine in the loop makes a hydrogen bond with the nucleotide $\gamma$-phosphate. This small change is transmitted to the coupling region where concerted domain movements result in the rotation of the LAH into the primed, pre-power stroke conformation. In myosin, ATP hydrolysis is a reversible equilibrium in the active site that occurs predominantly in the post-recovery $\mathrm{M} \uparrow \cdot \mathrm{ATP}$ state $^{38}$. This prevents unproductive release of hydrolysis products from a pre-recovery state $(\mathrm{M} \downarrow \cdot \mathrm{ATP})$. We have shown that $\mathrm{OM}$ specifically shifts the hydrolysis equilibrium towards products $\left(\mathrm{ADP} \cdot \mathrm{P}_{\mathrm{i}}\right)$. This in turn shifts the coupled equilibria $\left(K_{\mathrm{RS}}\right.$ and $K_{\mathrm{H}}$, Fig. 7) towards the primed LAH conformation $\left(\mathrm{M} \uparrow \cdot \mathrm{ADP} \cdot \mathrm{P}_{\mathrm{i}}\right)$ for rebinding to actin ${ }^{6}$.

The SW-II loop is contiguous with $\mathrm{N}$ terminus of the relay helix providing a link from the nucleotide pocket to the $\mathrm{SH} 1$ helix and the converter domain. A defining feature of the $\mathrm{M} \uparrow$ primed conformation is a kink in the relay helix around F489 of the human cMD. On the transition from $M \downarrow$ to $M \uparrow$, the bend in 
the relay helix is strongly coupled to the position of the SH1 helix and the converter domain ${ }^{13,33,37,39}$. The converter domain remains anchored to the relay helix as the converter-LAH undergoes its sweeping rotation. OM binds in the centre of the region that couples the $\mathrm{SH} 1$ helix, relay helix and converter domain and involves the key residues linking the $\mathrm{SH} 1$ helix to the relay helix (M493-C705 sulfhydryl H-bond) and the relay helix to the converter domain (E497-R712 salt bridge). OM binding shields these interactions from solvent and strengthens the links responsible for the concerted domain movement of the recovery stroke.

An extensive hydrophobic cluster involving Y501, I506, W508, F510, I511, F513, F709 and F764 is also involved in coupling the relay helix and loop with the SH1 helix and the converter domain $^{33}$. The E497-R712 salt bridge lies immediately below this aromatic cluster and is shielded from solvent on three sides by it; thus, OM binding completes the burial of the salt bridge in a hydrophobic pocket. This salt bridge is found in both the nearrigour and pre-power stroke conformation of the scallop myosin II MD structure, suggesting that it is essential to the coupling mechanism ${ }^{31}$. We have shown here that the drug-binding interactions are maintained through a $15^{\circ}$ rotation of the converter domain and LAH ( $\sim 25 \%$ of the recovery stroke), suggesting that the drug may stabilize this coupling region throughout the recovery stroke.

Both E497 and R712 have been identified as sites of cardiomyopathy-associated mutations: an E497D mutation results in a benign hypertrophic cardiomyopathy (HCM) and a R712L mutation is associated with a severe HCM phenotype and sudden cardiac death ${ }^{40,41}$. Three of the eight aromatic residues involved in the hydrophobic cluster are also sites associated with HCM (Y501, F513) and DCM (F764) mutations. Five of the sixteen residues that form the drug-binding cleft and interact with $\mathrm{OM}$ (M493, E497, V698, P710 and R712) are sites of missense mutations associated with human cardiac pathology. The clustering of cardiomyopathic mutations in this region correlates with the importance of these links to the communication pathway between the nucleotide state and priming of the LAH.

The Transducer is a structural element of the motor domain near the nucleotide-binding site that includes the last three strands of the central seven-stranded $\beta$-sheet of the $\mathrm{MD}^{13}$. Distortion of the $\beta$-sheet is essential for the domain movements needed to accommodate closure of the cleft between the upper and lower portions of the $50-\mathrm{K}$ domain on strong binding to actin ${ }^{13,20}$. That in turn reverses the rotation of the LAH leading to the power stroke (Fig. 7). The movements involve a twist of the last three strands $(\beta 5, \beta 6$ and $\beta 7)$ of the $\beta$-sheet, changes in the loops linking $\beta 6$ to $\beta 7$ ( $\beta$-bulge) and the HO helix to $\beta 5$, and Loop 1 (204-210). It has been suggested that the twist provides the structural basis for communication between the actin interface and the nucleotide pocket and may be involved in the $\gamma$-phosphate release $\mathrm{s}^{13,20,39,42}$. We have found that OM binding alters the conformation of the $\beta$-bulge and HO- $\beta 5$ loop and the twist of the $\beta 5, \beta 6$ and $\beta 7$ strands when compared with the Apo structure and between the two cMD conformations within the $\mathrm{OM}+$ structure. These structural changes are consistent with a mechanism, whereby $\mathrm{OM}$ accelerates the rate of $\mathrm{P}_{\mathrm{i}}$ release and the weak to strong actin-binding transition by facilitating $\beta$-sheet distortion.

We conclude that $\mathrm{OM}$ binding acts to stabilize the interactions within the coupling region. This region is central to the recovery stroke and the priming of the $\mathrm{CMD}$ for a productive contractile cycle. In addition, drug binding shifts the conformational equilibrium of the Transducer region to favour phosphate release. Together, these subtle changes alter the mechanical tuning of $\beta$-cardiac myosin producing changes in activity that in cardiac muscle will increase force production at the expense of speed of contraction. The candidate drugs from which OM was developed were originally identified by a high-throughput screen of a biochemically reconstituted contractile system ${ }^{3}$. That screen and subsequent selection of OM has identified a fundamentally important site for fine-tuning the motor activity of myosin.

\section{Methods}

Construction of the $\boldsymbol{\beta}$-cardiac myosin MD expression vector. A cDNA encoding the human $\beta$-cardiac muscle myosin HMM was kindly provided by James Sellers (National Heart, Lung, and Blood Institute (NHLBI)) from a clone isolated by Giovanni Cuda and Neil Epstein (NHLBI). Construction of the $\beta$ cardiac MD::GFP chimera is based on the skeletal muscle MD::GFP chimera $\left(\mathrm{S}_{795} \mathrm{GFP}\right)$ described in detail elsewhere ${ }^{9}$. A cDNA encoding residues 1-787 of the cardiac myosin motor domain was amplified with PCR using primers to insert a unique NotI site at the $5^{\prime}$ end of the open reading frame and a unique MluI site at the $3^{\prime}$ end. The cardiac MD sequence then replaced the skeletal muscle myosin MD in the shuttle vector $\mathrm{pSH}-\mathrm{S} 1_{795} \mathrm{GFP}$ (ref. 9). A four-residue linker (788-791) forms the fusion site to the GFP-coding sequence that comprise residues 5-238 of $A$. victoria GFP. The complete coding region was confirmed by sequencing. A FLAGtagged variant of chimeric protein was prepared by mutating the C-terminalcoding sequence of the GFP domain from DELYK to DYKDHD. This minimal FLAG tag sequence added a single additional residue to the protein, with no effect on protein activity or crystallization conditions ${ }^{43}$.

Adenovirus manipulation. Recombinant adenovirus DNA was prepared by homologous recombination of pSH-CaMD::GFP with the pAdEasyl vector in E. coli strain BJ5183 (refs 9,12,44). Colonies were selected for kanamycin resistance and plasmid DNA was characterized by restriction digestion. The recombinant virus DNA was transformed into E. coli $\mathrm{DH} 10 \mathrm{~B}$ cells, and the purified pAdCaMD DNA was linearized by digestion with PacI and transfected into human 293 cells (CRL 1573; American Type Culture Collection, Rockville, MD) for adenovirus packaging and amplification ${ }^{44}$. The human 293 cells are maintained in growth medium containing DMEM with $10 \%$ fetal bovine serum (FBS), $1 \mathrm{mM}$ sodium pyruvate and $0.5 \%$ gentamycin at $37^{\circ} \mathrm{C}$ and $5 \% \mathrm{CO}_{2}$. Cells were monitored using fluorescence microscopy for expression of the cMD::GFP fusion protein. The original human $\beta$-cardiac HMM clone encodes residues 1-1,138 of the MYH7 gene with a FLAG tag added on the C terminus $(1,139-1,146)$ of the S2 domain. The cHMM cDNA was cloned into the pShuttle-IRES-hrGFP-1 vector (Stratagene, LaJolla CA), and an AdcHMM-Flag virus was prepared and amplified for expression and purification of the CHMM protein in $\mathrm{C} 2 \mathrm{C} 12$ cells. The virus was expanded by infection of a large number of plates of confluent 293 cells at an multiplicity of infection of 3-5. The virus was harvested from the cells by cycles of freeze-thaw, and then it was purified by $\mathrm{CsCl}$ density sedimentation yielding final virus titres of $10^{10}-10^{11}$ plaque-forming units per $\mathrm{ml}$ (p.f.u. $\mathrm{ml}^{-1}$ ).

Muscle cell expression and purification of $\beta$-cardiac MD::GFP. Myoblasts of the mouse C2C12 cell line (CRL 1772; American Type Culture Collection) are maintained by seeding the cells at an initial density of $7.5 \times 10^{4}$ cells cm ${ }^{-2}$ in $90 \%$ DMEM, 10\% FBS and passaging the cells at less than $60 \%$ confluence ${ }^{9,12,45}$. Confluent $\mathrm{C} 2 \mathrm{C} 12$ myoblasts are infected with replication-defective recombinant adenovirus (Ad-CaMD::GFP) at $5 \times 10^{8}$ p.f.u. $\mathrm{ml}^{-1}$ in fusion medium $(89 \%$ DMEM, $10 \%$ horse serum, 1\% FBS) to induce differentiation. Expression of recombinant cMD::GFP is monitored by accumulation of GFP fluorescence in infected cells. Myocyte differentiation and fluorescence accumulation are monitored for the next 120-144 h when the cells are harvested. Cells are chilled, media is removed and the cell layer is rinsed with cold PBS. The cells are scraped into $0.5 \mathrm{ml}$ per dish of Triton extraction buffer: $100 \mathrm{mM} \mathrm{NaCl}, 0.5 \%$ Triton X-100, $10 \mathrm{mM}$ imidazole pH 7.0, $1 \mathrm{mM}$ dithiothreitol (DTT), $5 \mathrm{mM} \mathrm{MgATP}$ and Protease Inhibitor cocktail (Sigma, St Louis). The cell suspension is collected in an ice-cold Dounce homogenizer and lysed with 15 strokes of the A pestle. The cell debris in the whole-cell lysate is pelleted by centrifugation at $17,000 \mathrm{~g}$ for $15 \mathrm{~min}$ at $4{ }^{\circ} \mathrm{C}$. The Triton-soluble extract is fractionated by ammonium sulfate precipitation using two sequential steps of $0-30 \%$ saturation and $30-60 \%$ saturation. The CaMD::GFP precipitates between 30 and $60 \%$ saturation of ammonium sulfate.

The recovered pellet is dissolved in 1/10 the original volume of buffer and dialysed against $25 \mathrm{mM}$ imidazole, $\mathrm{pH} 7.0,1 \mathrm{mM}$ DTT for anion exchange chromatography of the cMD lacking the FLAG tag sequence, or into the same buffer without DTT for affinity purification of the FLAG-tagged cMD on M2 monoclonal antibody-Sepharose beads (Sigma). Anion exchange chromatography on a HR 5/5 Mono-Q column at $23^{\circ} \mathrm{C}$ (Pharmacia Biotech, Piscataway, NJ) was carried out as previously described ${ }^{9}$. The FLAG-tagged cMD was bound to an M2-Sepharose column, washed and eluted with $0.1 \mathrm{mg} \mathrm{ml}^{-1}$ FLAG peptide (Sigma). The FLAG-tagged human CHMM was purified in the same manner as the tagged cMD. Protein was concentrated and buffer-exchanged on Amicon Ultracel$10 \mathrm{~K}$ centrifugal filters (Millipore; Darmstadt, Germany), checked by SDS-PAGE (Supplementary Fig. 1), aliquoted and stored at $-80^{\circ} \mathrm{C}$. 
Motility and ATPase assays. An anti-GFP monoclonal antibody (3E6; Molecular Probes; Grand Island, NY) was bound to freshly prepared nitrocellulose-coated glass coverslips 9 . The cMD at $25 \mu \mathrm{g} \mathrm{ml}^{-1}$ was incubated with the surface and bound via the GFP domain as previously described. Skeletal muscle myosin was bound to the surfaces via an anti-S2 monoclonal antibody ${ }^{46,47}$. Recombinant human $\beta$-cardiac HMM at $40 \mu \mathrm{g} \mathrm{ml}^{-1}$ was bound directly to fresh nitrocellulose surfaces as described ${ }^{6,46}$. The surfaces were blocked with $1 \%$ bovine serum albumin in PBS for $5 \mathrm{~min}$. Motility is measured in a $12-\mu \mathrm{l}$ assay chamber in motility buffer ( $25 \mathrm{mM}$ Imidazole, $25 \mathrm{mM} \mathrm{KCl}, 4 \mathrm{mM} \mathrm{MgCl} 2,7.5 \mathrm{mM} \mathrm{Mg}^{2+}$ ATP, $0.5 \%$ methyl cellulose, $0.1 \mathrm{mg} \mathrm{ml}^{-1}$ glucose oxidase, $0.018 \mathrm{mg} \mathrm{ml}^{-1}$ catalase, $2.3 \mathrm{mg} \mathrm{ml}^{-1}$ glucose and $5 \mathrm{mM} 2$-mercaptoethanol, $\mathrm{pH}$ 7.6) containing $1 \mathrm{nM}$ phalloidinrhodamine-labelled actin. OM (CK-1827452) was purchased from Selleckchem.com (Boston, MA). To titrate the effect of the drug on motility, a 2.5-mM stock of OM in dimethylsulphoxide (DMSO) was serially diluted with DMSO before a final $1 / 200$ dilution into motility buffer with the rhodaminelabelled actin. The final $0.5 \%$ DMSO in the assay buffer had no effect on motility in the absence of drug. The chamber is observed with a temperature-controlled stage and objective set at $32{ }^{\circ} \mathrm{C}$ on an upright microscope with an image-intensified charge-coupled device camera capturing data to an acquisition computer at 5-30 f.p.s. dependent on assay parameters. Movement of actin filaments from 500 to 1,000 frames of continuous imaging is analysed with semiautomated filament tracking programmes ${ }^{12,46,48}$. The trajectory of every filament with a lifetime of at least 10 frames is determined; the instantaneous velocity of the filament moving along the trajectory, the filament length, the distance of continuous motion and the duration of pauses are tabulated. A weighted probability of the actin filament velocity for hundreds of events is fit to a Gaussian distribution and reported as a mean velocity and s.d. for each experimental condition.

Steady-state ATPase activity was measured as described previously ${ }^{49}$ Experiments were carried out in a buffer containing $4 \mathrm{mM}$ MOPS, $2 \mathrm{mM} \mathrm{MgCl}$ with or without $100 \mu \mathrm{M} \mathrm{OM}(0.5 \% \mathrm{DMSO}), \mathrm{pH}$ 7.2. The cMD at $0.06 \mu \mathrm{M}$ was used to measure the ATPase activity over a range of actin $(0-100 \mu \mathrm{M})$. The ATPase activity of the actin filaments alone was subtracted from the actomyosin data.

Crystallization and structure determination. Human $\beta$-cardiac MD::GFP protein $\left(1.5-2.0 \mathrm{mg} \mathrm{ml}^{-1}\right.$ ) in $25 \mathrm{mM}$ HEPES $\mathrm{pH} 7.0,5 \mathrm{mM}$ TCEP (Sigma) was screened against four Hampton Research (Aliso Viejo, CA) high-throughput crystallization screens using $0.2 \mu \mathrm{l}$ protein and $0.2 \mu \mathrm{l}$ mother liquor (ML) per drop equilibrated against $60 \mu \mathrm{l}$ of $\mathrm{ML}$ at room temperature. Five drops containing brightly fluorescent birefringent crystals were found. These initial conditions were refined and single crystals without drug (Apo) were grown from $10 \%$ Tacsimate, pH $6.0,14-15 \%$ PEG 3350, $10 \%$ glycerol, $0.2 \mathrm{mM} \mathrm{MgCl}_{2}$ and $5 \mathrm{mM}$ TCEP. Crystals with $\mathrm{OM}(\mathrm{OM}+)$ were grown in essentially the same condition with $125 \mu \mathrm{M}$ OM added to the protein drop ( $0.5 \%$ DMSO). The crystallization conditions proved to be a suitable cryoprotectant; therefore, crystals taken directly from the ML were fast-cooled in liquid nitrogen for X-ray analysis.

Single-crystal diffraction data for the Apo structure were collected on a Rigaku rotating anode X-ray generator $(\lambda=1.542 \AA)$ with a Raxis IV ++ detector at $100^{\circ} \mathrm{K}$. The $\mathrm{OM}+$ crystal diffraction data were collected at Brookhaven National Laboratory NSLS beamline X29A $(\lambda=1.075 \AA)$ with an ADSC QUANTUM 315 detector at $100^{\circ} \mathrm{K}$. Reflections were indexed, integrated and scaled with iMosflm and Aimless from the ccp4 package ${ }^{50,51}$. The Apo structure was determined first by molecular replacement with Phaser ${ }^{52}$ using residues $4-780$ of chicken skeletal muscle myosin subfragment 1 structure, PDB ID: $2 \mathrm{MYS}^{15}$, together with the structure of GFP, PDB ID: 2QLE ${ }^{53}$, as the search ensemble. The molecular replacement map clearly identified two motor domains associated with one GFP per domain. The helix linking the MD to the GFP was not included in the search models; however, new density corresponding to the helix was apparent in the initial maps. After building the linking helix into the density and joining the MD and GFP domains, the cMD and GFP sequence were built into the model with PHENIX Autobuild ${ }^{54}$. The structure was refined with PHENIX refine ${ }^{55}$ and iterative model building in $\mathrm{Coot}^{56}$. Only a very limited number of well-defined water molecules and two sulfate anions in the cMD $\beta$-phosphate-binding loop were included in the structure at this resolution. The statistics for favoured, allowed and outlier Ramachandran angles are $93.4 \%, 6.1 \%$ and $0.5 \%$, respectively. The Molprobity score for the refined structure is 2.16 and the coordinates are deposited as PDB ID: $4 \mathrm{P} 7 \mathrm{H}^{57,58}$

The $\mathrm{OM}+$ structure was solved by molecular replacement using the motor domain (2-708) of the Apo structure A chain and the helix-GFP domain as separate models in the search ensemble followed by PHENIX Autobuild. After limited initial refinement, unassigned density likely coming from the ligand was apparent. PHENIX LigandFit was used to search and dock OM into the map using Resolve $^{59}$. Ligand restraints for refinement were generated with $\mathrm{LLBOW}^{60}$. The structure was refined as with the Apo structure. The statistics for favoured, allowed and outlier Ramachandran angles are $95.3 \%, 4.2 \%$ and $0.5 \%$, respectively. The Molprobity score for the refined structure is 1.87 .

The Karplus and Diederichs $\mathrm{CC}^{\star}$ data and model quality-assessment tool were applied to both models and data sets to assess the high-resolution cutoff ${ }^{61}$ The Apo data set was truncated at $3.2 \AA$, with mean $(I / \sigma(I))=2$ and a $C^{\star}>0.88$ for the highest-resolution shell (3.3-3.2 $\AA$ ). The $\mathrm{CC}^{*}$ statistic for the $\mathrm{OM}+$ data is $>0.73$ for the highest-resolution shell (2.33-2.25 $\AA$ ), supporting the inclusion of the full data range in determination of the model ${ }^{62,63}$. Alternatively, applying traditional cutoff criteria of the mean $(I / \sigma(I)) \geq 2$ to the $\mathrm{OM}+$ data would make this a nominal $2.40-\AA$ resolution structure. The coordinates for the $\mathrm{OM}+$ structure are deposited as PDB entry 4PA0 (ref. 57). Data collection and refinement statistics are summarized in Table 1.

\section{References}

1. Lloyd-Jones, D. et al. Executive summary: heart disease and stroke statistics-2010 update: a report from the American Heart Association. Circulation 121, 948-954 (2010).

2. Teerlink, J. R. A novel approach to improve cardiac performance: cardiac myosin activators. Heart Fail. Rev. 14, 289-298 (2009).

3. Morgan, B. P. et al. Discovery of omecamtiv mecarbil the first, selective, small molecule activator of cardiac Myosin. ACS Med. Chem. Lett. 1, 472-477 (2010).

4. Malik, F. I. et al. Cardiac myosin activation: a potential therapeutic approach for systolic heart failure. Science 331, 1439-1443 (2011).

5. Shen, Y. T. et al. Improvement of cardiac function by a cardiac Myosin activator in conscious dogs with systolic heart failure. Circ. Heart Fail. 3, 522-527 (2010)

6. Liu, Y., White, H. D., Belknap, B., Winkelmann, D. A. \& Forgacs, E. Omecamtiv Mecarbil modulates the kinetic and motile properties of porcine beta-cardiac myosin. Biochemistry 54, 1963-1975 (2015).

7. Siemankowski, R. F., Wiseman, M. O. \& White, H. D. ADP dissociation from actomyosin subfragment 1 is sufficiently slow to limit the unloaded shortening velocity in vertebrate muscle. Proc. Natl Acad. Sci. USA 82, 658-662 (1985).

8. Wang, Y., Ajtai, K. \& Burghardt, T. P. Analytical comparison of natural and pharmaceutical ventricular myosin activators. Biochemistry 53, 5298-5306 (2014).

9. Chow, D., Srikakulam, R., Chen, Y. \& Winkelmann, D. A. Folding of the striated muscle myosin motor domain. J. Biol. Chem. 277, 36799-36807 (2002)

10. Liu, L., Srikakulam, R. \& Winkelmann, D. A. Unc45 activates Hsp90-dependent folding of the myosin motor domain. J. Biol. Chem. 283, 13185-13193 (2008).

11. Srikakulam, R. \& Winkelmann, D. A. Chaperone-mediated folding and assembly of myosin in striated muscle. J. Cell Sci. 117, 641-652 (2004).

12. Wang, Q., Moncman, C. L. \& Winkelmann, D. A. Mutations in the motor domain modulate myosin activity and myofibril organization. J. Cell Sci. 116, $4227-4238$ (2003).

13. Coureux, P. D., Sweeney, H. L. \& Houdusse, A. Three myosin V structures delineate essential features of chemo-mechanical transduction. EMBO J. 23, 4527-4537 (2004).

14. Klenchin, V., Deacon, J., Combs, A., Leinwand, L. \& Rayment, I. Cardiac human myosin S1dc, beta isofrom complexed with Mn-AMPPNP. PDB ID: 4DB1. 10.2210/pdb4db1/pdb (2012).

15. Rayment, I. et al. Three-dimensional structure of myosin subfragment-1: a molecular motor. Science 261, 50-58 (1993).

16. Risal, D., Gourinath, S., Himmel, D. M., Szent-Gyorgyi, A. G. \& Cohen, C. Myosin subfragment 1 structures reveal a partially bound nucleotide and a complex salt bridge that helps couple nucleotide and actin binding. Proc. Natl Acad. Sci. USA 101, 8930-8935 (2004).

17. Dominguez, R., Freyzon, Y., Trybus, K. M. \& Cohen, C. Crystal structure of a vertebrate smooth muscle myosin motor domain and its complex with the essential light chain: visualization of the pre-power stroke state. Cell 94, 559-571 (1998).

18. Houdusse, A., Kalabokis, V. N., Himmel, D., Szent-Gyorgyi, A. G. \& Cohen, C. Atomic structure of scallop myosin subfragment S1 complexed with MgADP: a novel conformation of the myosin head. Cell 97, 459-470 (1999).

19. Krissinel, E. \& Henrick, K. Inference of macromolecular assemblies from crystalline state. J. Mol. Biol. 372, 774-797 (2007).

20. Behrmann, E. et al. Structure of the rigor actin-tropomyosin-myosin complex Cell 150, 327-338 (2012).

21. Allingham, J. S., Smith, R. \& Rayment, I. The structural basis of blebbistatin inhibition and specificity for myosin II. Nat. Struct. Mol. Biol. 12, 378-379 (2005).

22. Kovacs, M., Toth, J., Hetenyi, C., Malnasi-Csizmadia, A. \& Sellers, J. R. Mechanism of blebbistatin inhibition of myosin II. J. Biol. Chem. 279, 35557-35563 (2004).

23. Fedorov, R. et al. The mechanism of pentabromopseudilin inhibition of myosin motor activity. Nat. Struct. Mol. Biol. 16, 80-88 (2009).

24. Preller, M., Chinthalapudi, K., Martin, R., Knolker, H. J. \& Manstein, D. J. Inhibition of Myosin ATPase activity by halogenated pseudilins: a structureactivity study. J. Med. Chem. 54, 3675-3685 (2011).

25. Radke, M. B. et al. Small molecule-mediated refolding and activation of myosin motor function. Elife 3, e01603 (2014).

26. Sweeney, H. L. \& Houdusse, A. Structural and functional insights into the Myosin motor mechanism. Annu. Rev. Biophys. 39, 539-557 (2010).

27. Changeux, J. P. 50 years of allosteric interactions: the twists and turns of the models. Nat. Rev. Mol. Cell Biol. 14, 819-829 (2013).

28. Greenberg, M. J., Shuman, H. \& Ostap, E. M. Inherent force-dependent properties of beta-cardiac myosin contribute to the force-velocity relationship of cardiac muscle. Biophys. J. 107, L41-L44 (2014). 
29. Spudich, J. A. Hypertrophic and dilated cardiomyopathy: four decades of basic research on muscle lead to potential therapeutic approaches to these devastating genetic diseases. Biophys. J. 106, 1236-1249 (2014).

30. Sun, Y. B., Lou, F. \& Irving, M. Calcium- and myosin-dependent changes in troponin structure during activation of heart muscle. J. Physiol. 587, 155-163 (2009).

31. Gourinath, S. et al. Crystal structure of scallop myosin S1 in the pre-power stroke state to 2.6 a resolution: flexibility and function in the head. Structure 11, 1621-1627 (2003)

32. Gulick, A. M., Bauer, C. B., Thoden, J. B. \& Rayment, I. X-ray structures of the MgADP, MgATPgammaS, and MgAMPPNP complexes of the Dictyostelium discoideum myosin motor domain. Biochemistry 36, 11619-11628 (1997).

33. Baumketner, A. The mechanism of the converter domain rotation in the recovery stroke of myosin motor protein. Proteins 80, 2701-2710 (2012).

34. Fischer, S., Windshugel, B., Horak, D., Holmes, K. C. \& Smith, J. C. Structural mechanism of the recovery stroke in the myosin molecular motor. Proc. Natl Acad. Sci. USA 102, 6873-6878 (2005).

35. Furch, M., Fujita-Becker, S., Geeves, M. A., Holmes, K. C. \& Manstein, D. J. Role of the salt-bridge between switch-1 and switch-2 of Dictyostelium myosin. J. Mol. Biol. 290, 797-809 (1999).

36. Kintses, B., Yang, Z. \& Malnasi-Csizmadia, A. Experimental investigation of the seesaw mechanism of the relay region that moves the myosin lever arm. J. Biol. Chem. 283, 34121-34128 (2008).

37. Koppole, S., Smith, J. C. \& Fischer, S. The structural coupling between ATPase activation and recovery stroke in the myosin II motor. Structure 15, 825-837 (2007).

38. Malnasi-Csizmadia, A. et al. Kinetic resolution of a conformational transition and the ATP hydrolysis step using relaxation methods with a Dictyostelium myosin II mutant containing a single tryptophan residue. Biochemistry 40, 12727-12737 (2001).

39. Sweeney, H. L. \& Houdusse, A. The motor mechanism of myosin V: insights for muscle contraction. Philos. Trans. R Soc. Lond. B Biol. Sci. 359, 1829-1841 (2004).

40. Arad, M. et al. Gene mutations in apical hypertrophic cardiomyopathy. Circulation 112, 2805-2811 (2005).

41. Sakthivel, S., Joseph, P. K., Tharakan, J. M., Vosberg, H. P. \& Rajamanickam, C. A novel missense mutation $(\mathrm{R} 712 \mathrm{~L})$ adjacent to the 'active thiol' region of the cardiac beta-myosin heavy chain gene causing hypertrophic cardiomyopathy in an Indian family. Hum. Mutat. 15, 298-299 (2000).

42. Holmes, K. C., Schroder, R. R., Sweeney, H. L. \& Houdusse, A. The structure of the rigor complex and its implications for the power stroke. Philos. Trans. $R$ Soc. Lond. B Biol. Sci. 359, 1819-1828 (2004).

43. Roosild, T. P., Castronovo, S. \& Choe, S. Structure of anti-FLAG M2 Fab domain and its use in the stabilization of engineered membrane proteins. Acta Crystallogr. Sect. F Struct. Biol. Cryst. Commun. 62, 835-839 (2006).

44. He, T. C. et al. A simplified system for generating recombinant adenoviruses. Proc. Natl Acad. Sci. USA 95, 2509-2514 (1998).

45. Kinose, F., Wang, S. X., Kidambi, U. S., Moncman, C. L. \& Winkelmann, D. A. Glycine 699 is pivotal for the motor activity of skeletal muscle myosin. J. Cell Biol. 134, 895-909 (1996).

46. Barua, B., Winkelmann, D. A., White, H. D. \& Hitchcock-DeGregori, S. E. Regulation of actin-myosin interaction by conserved periodic sites of tropomyosin. Proc. Natl Acad. Sci. USA 109, 18425-18430 (2012).

47. Winkelmann, D. A., Bourdieu, L., Ott, A., Kinose, F. \& Libchaber, A. Flexibility of myosin attachment to surfaces influences F-actin motion. Biophys. J. 68, 2444-2453 (1995).

48. Bourdieu, L., Magnasco, M. O., Winkelmann, D. A. \& Libchaber, A. Actin filaments on myosin beds: The velocity distribution. Phys. Rev. E 52, 6573-6579 (1995).

49. Forgacs, E. et al. Switch 1 mutation S217A converts myosin V into a low duty ratio motor. J. Biol. Chem. 284, 2138-2149 (2009).

50. Potterton, E., Briggs, P., Turkenburg, M. \& Dodson, E. A graphical user interface to the CCP4 program suite. Acta Crystallogr. D Biol. Crystallogr. 59, 1131-1137 (2003).

51. Evans, P. R. \& Murshudov, G. N. How good are my data and what is the resolution? Acta Crystallogr. D Biol. Crystallogr. 69, 1204-1214 (2013).

52. Adams, P. D. et al. PHENIX: a comprehensive Python-based system for macromolecular structure solution. Acta Crystallogr. D Biol. Crystallogr. 66, 213-221 (2010).
53. Shu, X. et al. An alternative excited-state proton transfer pathway in green fluorescent protein variant S205V. Protein Sci. 16, 2703-2710 (2007).

54. Terwilliger, T. C. et al. Iterative model building, structure refinement and density modification with the PHENIX AutoBuild wizard. Acta Crystallogr. D Biol. Crystallogr. 64, 61-69 (2008).

55. Afonine, P. V. et al. Towards automated crystallographic structure refinement with phenix.refine. Acta Crystallogr. D Biol. Crystallogr. 68, 352-367 (2012).

56. Emsley, P., Lohkamp, B., Scott, W. G. \& Cowtan, K. Features and developmen of Coot. Acta Crystallogr. D Biol. Crystallogr. 66, 486-501 (2010).

57. Berman, H. M. et al. The Protein Data Bank. Nucleic Acids Res. 28, 235-242 (2000).

58. Chen, V. B. et al. MolProbity: all-atom structure validation for macromolecular crystallography. Acta Crystallogr. D Biol. Crystallogr. 66, 12-21 (2010).

59. Terwilliger, T. C., Klei, H., Adams, P. D., Moriarty, N. W. \& Cohn, J. D. Automated ligand fitting by core-fragment fitting and extension into density. Acta Crystallogr. D Biol. Crystallogr. 62, 915-922 (2006).

60. Moriarty, N. W., Grosse-Kunstleve, R. W. \& Adams, P. D. electronic Ligand Builder and Optimization Workbench (eLBOW): a tool for ligand coordinate and restraint generation. Acta Crystallogr. D Biol. Crystallogr. 65, 1074-1080 (2009).

61. Karplus, P. A. \& Diederichs, K. Linking crystallographic model and data quality. Science 336, 1030-1033 (2012).

62. Diederichs, K. \& Karplus, P. A. Better models by discarding data? Acta Crystallogr. D Biol. Crystallogr. 69, 1215-1222 (2013).

63. Wang, J. \& Wing, R. A. Diamonds in the rough: a strong case for the inclusion of weak-intensity X-ray diffraction data. Acta Crystallogr. D Biol. Crystallogr. 70, 1491-1497 (2014).

64. Laskowski, R. A. \& Swindells, M. B. LigPlot + : multiple ligand-protein interaction diagrams for drug discovery. J. Chem. Inf. Model 51, 2778-2786 (2011).

\section{Acknowledgements}

We want to thank Lily Liu for technical assistance during the early stages of the work and Howard White for many helpful discussions on the interpretation of the results. We appreciate the assistance of Robert Sweet and Howard Robinson of Brookhaven National Laboratory NSLS beamline X29A. This work was supported by funding from the AHA (10GRNT4300022; 12BGIA12030120) and the NIH (1R56HL124284-01).

\section{Author contributions}

D.A.W. designed experiments, carried out the majority of the experimental work including the crystallography and wrote the manuscript. E.F. contributed to the kinetic assays, interpretation and integration of the kinetic data. M.T.M. and A.M.S. provided advice and guidance with the crystallography, and A.M.S. provided access to the X-ray sources and detectors. All authors discussed the results and contributed to the manuscript.

\section{Additional information}

Accession codes: Coordinates and structure factors for Apo cMD and $\mathrm{OM}+\mathrm{cMD}$ have been deposited in the Protein Data Bank under accession codes $4 \mathrm{P} 7 \mathrm{H}$ and $4 \mathrm{PA} 0$ respectively.

Supplementary Information accompanies this paper at http://www.nature.com/ naturecommunications

Competing financial interests: The authors declare no competing financial interests.

Reprints and permission information is available online at http://npg.nature.com/ reprintsandpermissions/

How to cite this article: Winkelmann, D. A. et al. Structural basis for drug-induced allosteric changes to human $\beta$-cardiac myosin motor activity. Nat. Commun. 6:7974 doi: $10.1038 /$ ncomms8974 (2015)

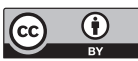

This work is licensed under a Creative Commons Attribution 4.0 International License. The images or other third party material in this article are included in the article's Creative Commons license, unless indicated otherwise in the credit line; if the material is not included under the Creative Commons license, users will need to obtain permission from the license holder to reproduce the material. To view a copy of this license, visit http://creativecommons.org/licenses/by/4.0/ 